\title{
RESULTS OF SYSTEMS INQUIRY
}

The following chart shows what equipment is used in the 59 institutions which answered the inquiry about language lab systems.

This chart will enable NALLD members to find out quickly where in the country a given system is being used. This should be useful for members who like to exchange information with colleagues who have systems similar to their own or for members who would like to collect first hand information on a given system before adopting it.

The present inquiry is the first one in a series designed to facilitate and encourage the exchange of information and ideas among NALLD members. It was conducted by Professor Emilio De Torre of Queens College, N.Y. who should be thanked here for his devotion to the cause, and congratulated for completing successfully such an onerous task.

The second inquiry in the series, on "holdings," is underway under the direction of Professor Joseph Sheehan of Georgetown University. It will enable members of NALLD to find out which institutions have programs in the less commonly taught languages.

Numbers used in chart identify institutions using the systems. See list below.

\begin{tabular}{l|l|l|l|l|l|l}
\hline \multirow{2}{*}{ SYSTEMS } & \multicolumn{3}{|c|}{ DIAL ACCESS } & \multicolumn{3}{c}{ TRADITIONAL } \\
\cline { 2 - 7 } & Open Reel & Cassette & Cartridge & Open Reel & Cassette & Cartridge \\
\hline Ampex & & & & 5,36 & & \\
\hline American Seating & & & & 24 & & \\
\hline Audijo Teaching Center & & & & & & \\
\hline A. V. Electronics & 54 & & & & & \\
\hline Advent & & 38 (stereo) & & & & \\
\hline
\end{tabular}




\begin{tabular}{|c|c|c|c|c|c|c|}
\hline \multirow{2}{*}{ SYSTEMS } & \multicolumn{3}{|c|}{ DIAL ACCESS } & \multicolumn{3}{|c|}{ TRADITIONAL } \\
\hline & Open Reel & Cassette & Cartridge & Open Reel & Cassette & Cartridge \\
\hline Bell & 37 & & 30 & & & \\
\hline Chester & $\begin{array}{l}19,27,30,33 \\
38,45(\mathrm{~s}), 49 \\
51,59\end{array}$ & & & & 3 & \\
\hline Cornely Elec. & & & & & & 48 \\
\hline Custom & & & & $9,36,37$ & 9 & \\
\hline Cybervox & & & & 29,31 & 5 & \\
\hline Dage Bell & 5 & & & & & \\
\hline DuKane & & & & 43 & & \\
\hline Educational Electronics & 55 & 55 & & 28 & & 28 \\
\hline Elektron & & & & 2 & & \\
\hline G. E. Labs & 34,35 & 、 & & 48 & & \\
\hline Instructomatic & 44 & & 44 & 46 & & \\
\hline Magnecord & 12 & & & & & \\
\hline Monitor & & & & 52,55 & & \\
\hline Own System & & & & 7 & & \\
\hline Raytheon & 2,4 & & 4,15 & $\begin{array}{l}10,15,22,26 \\
39,40,44\end{array}$ & 15,22 & 10 \\
\hline Rheem & & 37 & & $\begin{array}{l}8,14,16,17 \\
25,33,50\end{array}$ & 37 & \\
\hline R. C. A. & & & & $12,53,58$ & 12,58 & \\
\hline R. C. Merchant & & & & 23,49 & & \\
\hline Scotch & 26 & 26 & & & 26 & \\
\hline Sony & & 35 & & 2 & 32 & \\
\hline Tandberg & 7 & 2 & & $\begin{array}{l}6,18,20,32 \\
41,42,47 \\
\end{array}$ & & \\
\hline TEAC & & 45 & & & & \\
\hline Telex & 1,21 & & & & & \\
\hline Viking & 1,19 & & & $13,34,38,56$ & & \\
\hline Webster & & & & $11,21,57$ & 57 & \\
\hline Wollensak & & 7 & & & & \\
\hline
\end{tabular}


Here is a list of the institutions which responded to the first inquiry, with numbers used by which they are identified in the chart.

\author{
1. Albany SUNY \\ 2 - Alberta .U. of \\ 3 - Auburn U. \\ 4 - Barry C. \\ 5 - Belmont H. S. \\ $6+$ Boston U. \\ 7 - Brandeis U. \\ 8 - Brooklyn C. \\ 9 - California U. of \\ 10 - Capital U. \\ 11 - Claremont C. \\ 12 - Cleveland St. U. \\ 13 - Colorado U. of \\ 14 - Columbia U. \\ 15 - Eastbrook H. S. \\ 16 - Evansville U. of \\ 17 - Grants Pass H. S. \\ 18 - Harvard U. \\ 19 - Illinois U. of \\ 20 - Lebanon Valley C. \\ 21 - Massachusetts U. of \\ 22 - Marietta C. \\ 23 - McPherson C. \\ 24 - Michigan Tech. U. \\ 25 - Montana U. of \\ 26 - Mott C. C. \\ 27 - Nasson C. C. \\ 28 - Occidental C. \\ 29 - Ontario Inst. \\ 30 - Oswego SUNY
}

31 - Peddie School

32 - Pennsylvania U. of

33 - Queens C.

34 - Rhode Island U. of

35 - Rhode Island J. C.

36 - Ridgewood H. S.

37 - Rutgers St. U.

38 - Southern Conn. St. C.

39 - Southern Mission C.

40 - Staten Island C. C.

41 - Suffolk U.

42 - Sydney U. of

43 - Toronto U. of

44 - Townson St. C.

45 - Tufts U.

46 - Valley View H. S.

47 - Victoria U. (N.Z.)

48 - Virginia Poly Inst.

49 - Washington U. of

50 - Wayland Acad.

51 - Western Michigan U.

52 - Western Washington St. C.

53 - Westchester C. C.

54 - West Liberty St. C.

55 . Westminister Schools

56 . Wheaton C.

57 - Wilson C.

58 - Wisconsin U.

59 - Yale U. 